\title{
Intraoperative injuries during liver resection: analysis of 1,005 procedures
}

\author{
Michal Grat • Karolina Grzegorczyk • Zbigniew Lewandowski • Damian Sujecki • \\ Dawid Szwedowski • Adam Boltuc • Piotr Smoter • Oskar Kornasiewicz • \\ Marek Krawczyk
}

Received: 9 December 2010/Accepted: 30 May 2011/Published online: 14 June 2011

(c) The Author(s) 2011. This article is published with open access at Springerlink.com

\begin{abstract}
Purpose Risk of liver resection has been well investigated in many studies. However, the problem of intraoperative injuries is rarely mentioned. The aim of this study was to assess the incidence, the type, and management of intraoperative injuries during liver resection.

Methods A total of 1,005 liver resections between 2004 and 2009 were included in this retrospective investigation. We analyzed the incidence of intraoperative injuries, risk factors, and an impact on patients' clinical outcome.

Results The overall incidence of intraoperative injuries was $4.4 \%$ (44 of 1,005). Injuries of the diaphragm $(1.6 \%$, 16 of 1,005) and hepatocaval junction (1\%, 10 of 1,005) were the most frequent. In multivariate analysis, tumor recurrence $(p=0.0199)$ and tumor size $(p=0.0317)$ were the only independent risk factors for diaphragm injuries, whereas the extent of resection $(p=0.0007)$ was the only independent risk factor for caval or hepatic vein injuries. Injuries of the inferior vena cava or hepatic veins significantly increased perioperative mortality $(p=0.0005)$.

Conclusions Minor injuries causing no significant complications were the most frequent. However, prevention and proper management of the rare injuries of hepatocaval junction are essential to avoid increased mortality in major liver resections.
\end{abstract}

M. Grat $(\square) \cdot$ K. Grzegorczyk · D. Sujecki · D. Szwedowski · A. Boltuc - P. Smoter · O. Kornasiewicz · M. Krawczyk Department of General, Transplant and Liver Surgery, Medical University of Warsaw, 1A Banacha Street, 02-097 Warsaw, Poland

e-mail: michal.grat@gmail.com

Z. Lewandowski

Department of Epidemiology, Medical University of Warsaw, Oczki Street, 02-007 Warsaw, Poland
Keywords Liver resection - Mortality $\cdot$ Complications

\section{Introduction}

The role of liver resection in the treatment of both malignant and benign tumors is well established. Advancement in surgical techniques, diagnostic procedures, and perioperative management over the past decades has led to major improvement in patients' outcome [1]. The in-hospital mortality of $<3 \%$ and a complication rate ranging from 8.3 to $31 \%$ have been reported in many recent studies [2-6].

There are a large number of reports on factors influencing patients' outcome based on operative variables and tumor characteristics [4, 5, 7-10]. Complications following the resection of liver tumors include both procedure-related and general [11]. In the majority of studies, the most frequent events were cases of postoperative liver failure, perihepatic abscesses, biliary leakage, pleural effusion, and intraabdominal hemorrhage $[4,7-9,11-13]$. However, the incidence and the type of intraoperative injuries associated with liver resection are rarely mentioned. In a limited number of studies intraoperative injuries of the spleen, hepatic veins, intestines, and bile ducts are presented among other complications [8, 11, 12, 14].

Lehmann et al. [15] reported intraoperative injuries during surgical procedures accounting for $44 \%$ of admissions to the Intensive Care Units due to all iatrogenic events. Furthermore, intraoperative injuries of the abdominal arteries and veins are reported to be an increasingly important cause of all abdominal traumas [16-18]. In particular, the growing number of resections performed in the junction area between hepatic veins and the inferior vena cava has led to increased mortality and morbidity due to intraoperative laceration of these vessels [19-21]. The 
incidence of these events, most often complicated by massive bleeding or air embolism, reaches $\leq 15.5 \%$ in the most extensive and complex procedures [14].

Several risk factors for intraoperative injuries of the large vessels, the biliary tract, and other structures have been established. Previous abdominal surgeries, recurrence of the tumor, radiation therapy, and inflammatory changes were the most common [18, 22, 23].

The aim of this study was to establish the incidence, risk factors, and relevance of iatrogenic injuries during liver resection.

\section{Materials and methods}

A total of 1,005 consecutive liver resections for benign and malignant tumors in 912 patients between January 2004 and December 2009 were included in this retrospective investigation. The median age of the patients at the time of the procedure was 57 years (range 17-84 years). There were 393 males and 519 females. Of the total patients, 628 (68.9\%) had a history of previous abdominal surgeries.

Malignancy was the indication for resection in 829 $(82.5 \%)$ cases; $592(71.4 \%)$ of the malignant tumors were metastatic and 237 (28.6\%) were primary. Among metastases, 474 were of colorectal origin. The most frequent primary lesions were hepatocellular carcinoma (HCC 113 of $237,47.7 \%$ ), cancer of the extrahepatic bile ducts, including gallbladder (59 of 237, 24.9\%), and intrahepatic cholangiocarcinoma (CCC 52 of 237, 21.9\%). The numbers of cavernous hemangiomas, focal nodular hyperplasias, hepatic adenomas, and cysts were $73(41.5 \%), 58$ (33\%), 19 (10.8\%), and 7 (4\%) out of 176 benign tumors, respectively. Multiple lesions were found in 322 resected specimens. The median size of the tumor was $50 \mathrm{~mm}$ (range 5-370 mm).

Computed tomography and ultrasonography of the liver had been performed preoperatively in all cases. Magnetic resonance imaging was applied in those of uncertain diagnosis. Intraoperative ultrasonography of the liver was used during 133 procedures (13.2\%) and cholangiography in $82(8.2 \%)$.

Cirrhosis was found in the postoperative histopathological examination in 46 cases (4.6\%). According to ChildTurcotte-Pugh's classification, 8 patients were in class B while the remaining 38 belonged to class A. Out of 959 non-cirrhotic livers, steatosis of $\geq 30 \%$ hepatocytes was present in $77(8 \%)$.

In the majority of cases the abdominal cavity was explored by the bilateral subcostal incision with or without extension by the vertical midline incision. The method of liver tissue dissection was individually chosen by the operator and in most procedures concerned the use of ultrasonic dissector with bipolar coagulation or application of hemostatic clips in the cut surface. In the case of massive hemorrhage the methods employed to control the bleeding included the use of Pringle maneuver, selective or total vascular exclusion of the liver whenever necessary. Venovenous bypass was not applied in those cases. No other methods were used to anticipate the bleeding. The technique of liver resection did not significantly change over the studied period.

All procedures were performed by a group of surgeons from a single department. In order to analyze humanrelated factors they were arbitrarily divided into two groups according to their experience in the field of hepatobiliary surgery. Surgeons were considered senior if both of the following criteria were met: 10 years of experience in hepatobiliary surgery, more than 50 liver resections performed. The second variable included the number of liver resections performed 6 months prior to the analyzed procedure.

There were $427(42.5 \%)$ anatomical resections and 395 (39.3\%) non-anatomical ones. In $123(18.2 \%)$ cases the resection was both anatomical and non-anatomical. Total $656(65.3 \%)$ resections were unilateral and 349 (34.7\%) bilateral. Major resections defined as a removal of three or more Couinaud segments were performed in 699 (69.6\%) cases. Pringle maneuver was applied during 264 (26.3\%) resective surgeries. The removal of all hepatic lesions was achieved in $920(91.5 \%)$ procedures.

In order to achieve oncological radicality, 33 resections were extended to adjacent structures. These included diaphragm in 28 patients, right adrenal gland in 3 , and both in 1. Hilar lymphadenectomy was performed in 60 surgeries. Roux en Y loop hepaticojejunostomy with the resection of extrahepatic bile ducts was required in 45 cases. Combined surgical treatment with cryo- or radiofrequency ablation have associated $72(7.2 \%)$ resections.

The data in operative charts were reviewed retrospectively. Each case of inadvertent injury of abdominal organs was classified as an intraoperative injury. The incidence, types of injuries, and techniques of surgical management were analyzed.

Fisher's exact test was used to examine the relation between categorical variables. Forward stepwise logistic regression was applied for multivariate analysis. Patients' demographics, history of previous surgeries, tumor characteristics, and details of the procedure were considered in both uni- and multivariate analyses of risk factors for intraoperative injuries. A categorical variable defining individual experience of the surgeon was included in those multivariate models to adjust for its possible confounding role. Odds ratio (OR) values are given in brackets with exact $95 \%$ confidence intervals (95\% CIs). Each death during postoperative hospital stay accounted for the 
mortality rate. The impact of intraoperative injuries on the risk of postoperative complications and mortality was analyzed in bivariate models. We considered factors as independent if statistical significance was reached in bivariate analyses. The following factors were taken into consideration as possible confounders: presence of cirrhosis, grade of macrovacuolar liver steatosis, patient's age, extent of resection in terms of the number of resected Couinaud segments, and malignancy as an indication for surgery. A $p$ value of $<0.05$ was considered statistically significant. SAS system (SAS STAT User's Guide, SAS Institute, NC, 2009) was used in statistical analyses.

\section{Results}

A total of 50 intraoperative injuries during 44 liver resections $(4.4 \%)$ were recorded. Multiple injuries were found in four procedures. Types and incidence of intraoperative injuries are presented in Table 1.

All intraoperative injuries of the diaphragm occurred during the mobilization of the right lobe. A concomitant injury of the pericardium was present in one case. All of the 16 cases resulted in right-side pneumothorax and were treated with sutures and aspiration of air from the right pleural space. Excessive intraoperative hemorrhage as a result of diaphragm injury was observed in one patient. A single case of associated pericardial injury was managed with vascular sutures. There were no specific postoperative complications related to the injury of the diaphragm.

Seven injuries of the hepatic veins and three of the retrohepatic vena cava were found, all of them including partial lacerations. Each intraoperative injury of these large veins occurred during the preparation of the tumor in the liver tissue. The data on seven intraoperative injuries of the hepatic veins, repair procedures, the type of resection, and associated complications are shown in Table 2. One patient died during the surgery due to massive blood loss from the damaged right hepatic vein. Injuries of the inferior vena cava were treated with venorrhaphy; there were no

Table 1 Types and incidence of intraoperative injuries

\begin{tabular}{lll}
\hline Type of injury & Incidence $(\%)$ & $95 \%$ CI \\
\hline Overall & 4.38 & $3.20-5.83$ \\
Diaphragm & 1.59 & $0.91-2.57$ \\
Caval and hepatic veins & 1.00 & $0.48-1.82$ \\
Spleen & 0.70 & $0.28-1.43$ \\
Intestines & 0.70 & $0.28-1.43$ \\
Extrahepatic bile ducts & 0.50 & $0.16-1.16$ \\
Portal vein & 0.40 & $0.11-1.02$ \\
\hline
\end{tabular}

intraoperative deaths in this group and recovery of the patients was uneventful.

Three out of seven intraoperative injuries of the spleen required splenectomy due to the inability to stop the bleeding. The rest were treated with a combination of compression, oxycelulose application, and argon coagulation. No specific postoperative complications were related to the splenic injuries.

Intraoperative injuries of the small intestine (5) and colon (2) occurred prior to the resection of the liver tissue due to peritoneal adhesions. A history of previous abdominal surgeries had been noted in all of these patients. Two intraoperative perforations of the jejunum were managed with a partial resection with end-to-end intestine anastomosis and the rest with a double layer of single sutures.

In four cases intraoperative injury of the common bile duct occurred during the preparation of the hepatoduodenal ligament and in one during cholecystectomy. Primary repair included Roux-en-Y hepatojejunostomy in three patients and simple sutures in the remaining two. No postoperative biliary complications were noted in this group of patients.

In all the cases the portal vein was injured during the preparation of the structures of the liver hilum and it was repaired with vascular sutures. Postoperative ultrasonography revealed stenosis of the portal vein in one case resulting in liver ischemia. However, biochemical manifestation of liver function impairment had resolved over the postoperative period. One patient with concomitant injury of the hepatic artery, the common bile duct, and the right hepatic vein died 2 days after the surgery due to multiorgan failure. The management of this particular portal vein injury included end-to-end anastomosis of the completely lacerated vessel. The others were treated with continuous sutures of the partially injured veins.

Univariate analysis of risk factors for intraoperative injuries is presented in Table 3. In multivariate analysis the operation of the tumor recurrence and tumor size were the only independent risk factors for diaphragm injuries, whereas the extent of the resection was the only independent risk factor for caval or hepatic vein injuries (Table 4).

Overall in-hospital morbidity and mortality rates were $22.1 \%$ (222 of 1,005) and $1.4 \%$ (14 of 1,005), respectively. The types of postoperative complications and causes of death are presented in Table 5. One patient committed suicide in the postoperative period and was not included in the overall mortality rate.

The overall morbidity rate reached $43.2 \%$ (19 of 44 ) in patients with intraoperative injuries compared to $21.1 \%$ (203 of 961) in the other patients, $p=0.0013$ (OR $=2.84$, 95\% CI 1.53-5.26). Intraoperative injuries in general and caval or hepatic vein injuries were associated with increased in-hospital mortality (Table 6). 
Table 2 Intraoperative injuries of the hepatic veins

\begin{tabular}{llll}
\hline Hepatic vein(s) & Type of resection & Repair procedure & Complications \\
\hline Right & ERH & Venorrhaphy & $\begin{array}{c}\text { Death 2 days after surgery due } \\
\text { to multiorgan failure }\end{array}$ \\
Right, middle & ERH & Venorrhaphy & Cardiac arrest \\
Middle & ERH & Venorrhaphy & Air embolism \\
Middle & ELH & Venorrhaphy and TVE & Massive bleeding \\
Right & RH & Venorrhaphy & Operative death \\
Left & LH & Venorrhaphy & Massive bleeding \\
Right & RH & Venorrhaphy and TVE & Massive bleeding \\
\hline
\end{tabular}

$R H$ right hemihepatectomy, $L H$ left hemihepatectomy, $E R H$ extended right hemihepatectomy, $E L H$ extended left hemihepatectomy, $T V E$ total vascular exclusion

In each of the bivariate models intraoperative injuries in general, as well as injuries of the hepatocaval junction and liver hili were significant risk factors for postoperative morbidity. Regarding postoperative mortality, injuries in general and injuries of the hepatocaval junction reached the level of statistical significance in each case.

\section{Discussion}

Injuries of the diaphragm with no significant effect on patient's outcome were observed to be the most frequent in the studied group. All of these events were diagnosed and treated intraoperatively. A single case of excessive hemorrhage from the diaphragm was probably induced by coagulopathy in a patient with cirrhosis. No specific postoperative complications after the injury of the diaphragm were observed. Nevertheless, the injuries of the diaphragm were obviously associated with the opening of the right pleural space and, in one case, with the opening of the pericardium. Therefore, an increased risk of postoperative respiratory complications including infections cannot be excluded. In bivariate models diaphragm injuries were not an independent risk factor for increased morbidity; however, this fact may be related to an insufficient number of injuries observed. The operation for the recurrence of the tumor significantly increased the risk of this type of injury. This might have been due to technical difficulties caused by distorted anatomy in the previously resected area as described elsewhere [18]. Moreover, the risk of diaphragm injury increased slightly with tumor size.

On the contrary, the majority of hepatic veins or the inferior vena cava injuries resulted in potentially lethal operative complications, including massive bleeding and air embolism. In each case, venorraphy of the injured vessel with the suturing of the junction between the inferior vena cava and the hepatic vein was possible because it had been planned otherwise in the further course of the
Table 3 Univariate analysis of factors associated with intraoperative injuries

$N S$ nonsignificant

a All intestinal injuries occurred in males

\begin{tabular}{|c|c|c|c|c|c|c|}
\hline & $\begin{array}{l}\text { Overall } \\
\text { injuries } \\
p \text {-value }\end{array}$ & $\begin{array}{l}\text { Diaphragm } \\
\text { injuries } \\
p \text {-value }\end{array}$ & $\begin{array}{l}\text { Intestinal } \\
\text { injuries } \\
p \text {-value }\end{array}$ & $\begin{array}{l}\text { Caval and } \\
\text { hepatic } \\
\text { veins injuries } \\
p \text {-value }\end{array}$ & $\begin{array}{l}\text { Hilar } \\
\text { structures } \\
\text { injuries } \\
p \text {-value }\end{array}$ & $\begin{array}{l}\text { Spleen } \\
\text { injuries } \\
p \text {-value }\end{array}$ \\
\hline Male gender & 0.02 & NS & $-^{\mathrm{a}}$ & 0.038 & NS & NS \\
\hline \multicolumn{7}{|l|}{ Tumor } \\
\hline Malignant & NS & NS & NS & NS & NS & NS \\
\hline Size & NS & NS & NS & 0.014 & NS & NS \\
\hline Bilateral & NS & NS & NS & NS & NS & NS \\
\hline $\begin{array}{l}\text { Previous abdominal } \\
\text { procedures }\end{array}$ & NS & NS & 0.05 & NS & NS & NS \\
\hline Colorectal resection & NS & NS & 0.048 & NS & NS & NS \\
\hline \multicolumn{7}{|l|}{ Procedure } \\
\hline No. of resected segments & NS & NS & NS & 0.004 & NS & NS \\
\hline Bilateral resection & NS & NS & NS & NS & NS & NS \\
\hline Additional procedures & NS & NS & NS & NS & NS & 0.007 \\
\hline Tumor recurrence & NS & 0.028 & NS & NS & NS & NS \\
\hline
\end{tabular}


Table 4 Multivariate analysis of risk factors for specific intraoperative injuries

\begin{tabular}{|c|c|c|c|c|}
\hline Risk factor & Injury & OR & $95 \% \mathrm{CI}$ & $p$-value \\
\hline Tumor recurrence & Diaphragm & 5.99 & $1.69-21.21$ & 0.0199 \\
\hline Tumor size & Diaphragm & $1.12^{\mathrm{a}}$ & $1.02-1.22$ & 0.0317 \\
\hline Non-senior surgeon & Diaphragm & 3.45 & $0.92-12.99$ & 0.0520 \\
\hline No. of resected segments & IVC or hepatic veins & 2.82 & $1.37-5.80$ & 0.0007 \\
\hline
\end{tabular}

IVC inferior vena cava

${ }^{\text {a }}$ Per $1 \mathrm{~cm}$ increase

Table 5 Complications and causes of death

\begin{tabular}{lc}
\hline & Number $(\%)$ \\
\hline Complications & $222(22.1)$ \\
Transient liver insufficiency & $50(5.0)$ \\
Uninfected fluid collection & $30(3.0)$ \\
Biliary fistula & $30(3.0)$ \\
Ascites & $23(2.3)$ \\
Prolonged serose leak ${ }^{\mathrm{a}}$ & $21(2.1)$ \\
Bleeding/hematoma & $21(2.1)$ \\
Need for reoperation & $16(1.6)$ \\
Wound infection & $11(1.1)$ \\
Pleural effusion & $11(1.1)$ \\
Fever of unknown origin & $8(0.8)$ \\
Multiorgan failure & $6(0.6)$ \\
Renal failure & $5(0.5)$ \\
Perihepatic abscess & $5(0.5)$ \\
Atrial fibrillation & $3(0.3)$ \\
Eventration & $3(0.3)$ \\
Pneumothorax & $3(0.3)$ \\
Ischemic stroke & $3(0.3)$ \\
Pneumonia & $3(0.3)$ \\
Pancreatitis & $2(0.2)$ \\
Hepatic vein thrombosis & $2(0.2)$ \\
Enteric fistula & $2(0.2)$ \\
Mortality & $14(1.4)$ \\
Liver failure & $7(0.7)$ \\
Bleeding (postoperative) & $3(0.3)$ \\
Bleeding (intraoperative) & $1(0.1)$ \\
Sepsis & $1(0.1)$ \\
Multiorgan failure & $1(0.1)$ \\
Respiratory insufficiency & $1(0.1)$ \\
\hline
\end{tabular}

${ }^{a}$ Discharged from hospital without removal of drainage

operation. Although the repair of the injured veins has been claimed to increase the risk of postoperative thrombosis and thromboembolic complications compared with ligation of the vessel [24], it has not been proved in recent studies [25]. We have not observed such complications in patients with injuries of the hepatocaval junction.
The risk of caval or hepatic vein injury increased with the number of resected segments. This is a result of the interest in radical oncologic resection of tumors located near the hepatocaval junction in most cases requiring extensive procedures.

In one of the cases, both the right and the middle hepatic vein were injured with severe bleeding that led to cardiac arrest. This surgery was indicated by an extremely large HCC $(190 \mathrm{~mm})$ in the non-cirrhotic liver and the patient had no coexisting liver disease. Laceration of the right hepatic vein was successfully managed with an application of Pringle maneuver and sutures. However, subsequent injury of the middle hepatic vein occurred in the further course of the operation with massive hemorrhage and cardiac arrest.

Air embolism as a result of the middle hepatic vein injury occurred in one patient. This vessel was accidentally cut during the preparation of the middle hepatic vein in the liver parenchyma in the course of right extended hemihepatectomy. In another case, hemorrhage from the right hepatic vein led to the patient's death during the right hemihepatectomy for a giant tumor $(>100 \mathrm{~mm})$. During this surgery another injury of the same vessel occurred, consequent to cardiac massage through the diaphragm after the suturing of the former laceration.

One postoperative death in the course of multiorgan failure was noted in a patient with multiple injuries of the right hepatic vein, the portal vein, the hepatic artery, and the left hepatic duct. The patient had no preexisting liver disease and underwent right extended hemihepatectomy for large centrally located CCC infiltrating the hepatoduodenal ligament. The structures of the liver hili were injured due to the manipulation of the tumor during the preparation of the hepatoduodenal ligament. The right hepatic vein was lacerated in the final stage of the resection.

Stenosis of the portal vein was found in only one patient with intraoperative injury of this vessel. This patient was operated for a large hilar CCC situated close to the bifurcation of the portal vein. Partial laceration of the right branch of the portal vein occurred during the preparation of the tumor in the liver hilum and was managed with continuous suture. However, liver ischemia due to postoperative stenosis resulted only in prolonged normalization of serum aspartate and 
Table 6 Univariate analysis of the influence of intraoperative injuries on postoperative morbidity and mortality

\begin{tabular}{|c|c|c|c|c|}
\hline \multirow[t]{2}{*}{ Type of injury } & \multicolumn{2}{|l|}{ Morbidity } & \multicolumn{2}{|l|}{ Mortality } \\
\hline & OR $(95 \% \mathrm{CI})$ & $p$-value & OR $(95 \% \mathrm{CI})$ & $p$-value \\
\hline Overall & $2.84(1.53-5.26)$ & 0.0009 & $5.79(1.57-21.31)$ & 0.0083 \\
\hline Diaphragm & $2.80(1.03-7.61)$ & 0.0434 & - & - \\
\hline Caval and hepatic veins & $8.47(2.17-33.01)$ & 0.0021 & $18.89(3.65-97.67)$ & 0.0005 \\
\hline Spleen & - & - & - & - \\
\hline Intestines & $4.77(1.06-21.47)$ & 0.0418 & - & - \\
\hline Hilar structures & $5.99(1.42-25.25)$ & 0.0148 & $10.03(1.16-87.04)$ & 0.0365 \\
\hline
\end{tabular}

alanine transaminases activities. No clinical signs of liver insufficiency were observed in this patient. The remaining cases had no relevant influence on patients' outcome. Furthermore, postoperative bleeding from the repaired structure was not found in any of those patients. However, proper management of vascular injuries is necessary to avoid longterm complications of thromboembolic nature.

Immediate diagnosis and repair of the bile duct injuries are of basic importance in order to prevent complications in the future. In the present investigation, bile duct injuries were successfully repaired during primary surgery with no long-term complications observed. The rate of $0.5 \%$ was similar to those reported for iatrogenic biliary injuries during laparoscopic cholecystectomies [26-29]. Postoperative bile leakage occurred after $3 \%$ of procedures; however, none of those complications were related to the injuries of the extrahepatic bile ducts. Majority of these leaks originated from the resection surface.

Almost half of the splenic injuries required splenectomy due to excessive bleeding. Hemostasis with argon beamer, compression, and oxycellulose application was successful in minor injuries involving the splenic capsule. The influence of splenectomy due to intraoperative injuries on the morbidity rate and specific complications remains unclear [30, 31]. In this investigation, patients undergoing forced splenectomy did not develop any postoperative surgical complications. Any additional procedure involving the left upper quadrant of the abdomen may increase the risk of splenic injury. Therefore, in this study, intraoperative injuries of the spleen were more frequent if an extrahepatic procedure was performed.

Dense peritoneal adhesions led to intraoperative bowel injury in $<1 \%$ of the procedures. Partial resection of the damaged intestine or simple suturing was sufficient in all cases, preventing gastrointestinal or infectious complications.

The impact of the experience of the operator on the risk of intraoperative injuries in general and on the risk of particular injuries was on the verge of statistical significance. Although the majority of surgeries were performed by experienced surgeons, the level of significance may not have been reached because of the small number of procedures performed by less experienced operators. The number of procedures performed 6 months prior to each analyzed surgery did not have any impact on the rate of intraoperative injuries.

In this study, the abdominal cavity was opened by bilateral subcostal incision, extended to Mercedes-Benz incision whenever required. However, for most extensive hepatic resections inverted- $\vartheta$ approach with or without thoracotomy is sometimes advocated. In our opinion, the Mercedes-Benz approach is sufficient to visualize the area around hepatocaval junction. In $>1,000$ of presented procedures thoracotomy was never required to achieve a good operating view. Moreover, the rate of hepatocaval junction injuries did not vary between the bilateral subcostal approach and the Mercedes-Benz approach.

According to univariate analyses, the risk of developing postoperative complications was increased in intraoperative injuries in general and in each particular type of injury except splenic. Moreover, none of the patients suffering from intraoperive splenic injuries had postoperative complications. Unfortunately, small absolute numbers of specific injuries precluded standard multivariate analyses. Therefore, in order to avoid artifact results and to eliminate possible confounders we performed a set of bivariate analyses for each injury regarding perioperative mortality and morbidity.

Intraoperative injuries in general were a risk factor for postoperative morbidity independently of coexisting liver cirrhosis, the degree of macrovacuolar liver steatosis, the patient's age, malignant indication, and the extent of the procedure. More specifically, injuries of the hepatocaval junction and structures localized in the liver hilum were independent risk factors for the occurrence of postoperative complications as well. The impact of other injuries, except splenic, was on the verge of statistical significance.

The significant increase in postoperative morbidity in patients with intraoperative laceration of the hepatocaval junction is most certainly the result of massive hemorrhage from the injured vessels, multiplied requirement for transfusions, and prolonged duration of the surgery. The amount of blood loss from the injured veins until the localization and clamping of the vessel is in the majority of cases mostly extensive. 
The mortality rate of $1.4 \%$ was comparable to other reports. Intraoperative injuries were an independent risk factor for in-hospital mortality. Regarding specific types, only injuries of the hepatocaval junction were an independent risk factor for mortality. Both intraoperative and postoperative deaths are reported in the present study and each of those was probably the result of extensive blood loss from the injured vessel and related general complications.

Postoperative bleeding was responsible for 3 of 14 inhospital deaths. In the first patient the removal of all lesions was impossible due to the insufficient volume of the potential remnant of the liver; therefore, the tumor was treated with radiofrequency ablation. In this case bleeding originated from the unresected tumor and the patient underwent a consequent surgery on the same day. Unfortunately, the patient died a few hours after the secondary operation. In the second patient, attempts to stop the hemorrhage in three subsequent surgeries were not successful. Moreover, the origin of postoperative bleeding was not identified. In the third patient, postoperative bleeding resulted in a large splenic hematoma. Secondary surgery was performed and the patient was lost intraoperatively. This case was potentially associated with unrecognized splenic injury.

Open Access This article is distributed under the terms of the Creative Commons Attribution Noncommercial License which permits any noncommercial use, distribution, and reproduction in any medium, provided the original author(s) and source are credited.

\section{References}

1. Fortner JG, Blumgart LH. A historic perspective of liver surgery for tumors at the end of the millenium. J Am Coll Surg 2001;193:210-222

2. Virani S, Michaelson JS, Hutter MM, et al. Morbidity and mortality after liver resection: results of the patients safety in surgery study. J Am Coll Surg 2007;204:1284-1292

3. Ferrero A, Polastri R, Muratore A, Zorzi D, Capussotti L. Extensive resections for colorectal liver metastases. J Hepatobiliary Pancreat Surg 2004;11:92-96

4. Ohlsson B, Stenram U, Tranberg KG. Resection of colorectal liver metastases: 25-year experience. World J Surg 1998; 22:268-276

5. Belli G, D'Agostino A, Ciciliano F, Fantini C, Russolillo N, Belli A. Liver resection for hepatic metastases: 15 years of experience. J Hepatobiliary Pancreat Surg 2002;9:607-613

6. Ibrahim S, Chen CL, Wang SH, et al. Liver resection for benign liver tumors: indications and outcome. Am J Surg 2007; 193:5-9

7. Benzoni E, Cojutti A, Lorenzin D, et al. Liver resective surgery: a multivariate analysis of postoperative outcome and complication. Langenbecks Arch Surg 2007;392:45-54

8. Mutsaerts EL, van Ruth S, Zoetmulder FA, Rutgers EJ, Hart AA, van Coevorden F. Prognostic factors and evaluation of surgical management of hepatic metastases from colorectal origin: a 10-year single institute experience. J Gastrointest Surg 2005;9:178-186
9. Nagino M, Kamiya J, Uesaka K, et al. Complications of hepatectomy for hilar cholangiocarcinoma. World J Surg 2001;25:1277-1283

10. Konstadoulakis MM, Roayaie S, Gomatos IP, et al. Fifteen-year, single-center experience with the surgical management of intrahepatic cholangiocarcinoma: operative results and long-term outcome. Surgery 2008;143:366-374

11. Fan MH, Chang AE. Resection of liver tumors: technical aspects. Surg Oncol 2002;10:139-152

12. Yoon SS, Charny CK, Fong Y, et al. Diagnosis, management, and outcomes of 115 patients with hepatic hemangioma. J Am Coll Surg 2003;197:392-402

13. Nanashima A, Sumida Y, Abo T, Nagayasu T, Sawai T. Usefulness and application of liver hanging maneuver for anatomical liver resections. World J Surg 2008;32:2070-2076

14. Zhou W, Li A, Pan Z, et al. Selective hepatic vascular exclusion and Pringle maneuver: a comparative study in liver resection. Eur J Surg Oncol 2008;34:49-54

15. Lehmann LS, Puopolo AL, Shaykevich S, Brennan TA. Iatrogenic events resulting in intensive care admission: frequency, cause, and disclosure to patients and institutions. Am J Med 2005; 118:409-413

16. Nehler MR, Taylor LM Jr, Porter JM. Iatrogenic vascular trauma. Semin Vasc Surg 1998;11:283-293

17. Lazarides MK, Arvanitis DP, Liatas AC, Dayantas JN. Iatrogenic and noniatrogenic arterial trauma: a comparative study. Eur J Surg 1991;157:17-20

18. Oderich GS, Panneton JM, Hofer J, et al. Iatrogenic operative injuries of abdominal and pelvic veins: a potentially lethal complication. J Vasc Surg 2004;39:931-936

19. Smyrniotis V, Arkadopoulos N, Kehagias D, et al. Liver resection with repair of major hepatic veins. Am J Surg 2002;183:58-61

20. MacKenzie S, Dixon E, Bathe O, Sutherland F. Intermittent hepatic vein total vascular exclusion during liver resection: anatomic and clinical studies. J Gastrointest Surg 2005;9:658-666

21. Scheele J, Stang R, Altendorf-Hofmann A, Paul M. Resection of colorectal liver metastases. World J Surg 1995;19:59-71

22. Oktar GL. Iatrogenic major venous injuries incurred during cancer surgery. Surg Today 2007;37:366-369

23. Strasberg SM. Avoidance of biliary injury during laparoscopic cholecystectomy. J Hepatobiliary Pancreat Surg 2002;9:543-547

24. Timberlake GA, Kerstein MD. Venous injury: to repair or ligate, the dilemma revisited. Am Surg 1995;61:139-145

25. Quan RW, Gillespie DL, Stuart RP, Chang AS, Whittaker DR, Fox CJ. The effect of vein repair on the risk of venous thromboembolic events: a review of more then 100 traumatic military venous injuries. J Vasc Surg 2008;47:571-577

26. Deziel DJ, Millikan KW, Economou SG, Doolas A, Ko ST, Airan MC. Complications of laparoscopic cholecystectomy: a national survey of 4, 292 hospitals and an analysis of 77, 604 cases. Am J Surg 1993;165:9-14

27. Fletcher DR, Hobbs MS, Tan P, et al. Complications of cholecystectomy: risks of the laparoscopic approach and protective effects of operative cholangiography: a population-based study. Ann Surg 1999;229:449-457

28. MacFadyen BV Jr, Vecchio R, Ricardo AE, Mathis CR. Bile duct injury after laparoscopic cholecystectomy. The United States experience. Surg Endosc 1998;12:315-321

29. Calvete J, Sabater L, Camps B, et al. Bile duct injury during laparoscopic cholecystectomy: myth or reality of the learning curve. Surg Endosc 2000;14:608-611

30. Coon WW. Iatrogenic splenic injury. Am J Surg 1990;159:585-588

31. Eaton MA, Valentine J, Jackson MR, Modrall G, Clagett P. Incidental splenic injury during abdominal vascular surgery: a case-controlled analysis. J Am Coll Surg 2000;190:58-64 